IUCM95-036

\title{
Thermodynamics of Quantum Hall Ferromagnets
}

\author{
Marcus Kasner ${ }^{\dagger}$ and A. H. MacDonald \\ Dept. of Physics, Indiana University, Swain Hall West 117, Bloomington, IN 47405, USA
}

(October 18, 2018)

\begin{abstract}
The two-dimensional interacting electron gas at Landau level filling factor $\nu=1$ and temperature $T=0$ is a strong ferromagnet; all spins are completely aligned by arbitrarily weak Zeeman coupling. We report on a theoretical study of its thermodynamic properties using a many-body perturbation theory approach and concentrating on the recently measured temperature dependence of the spin magnetization. We discuss the interplay of collective and single-particle aspects of the physics and the opportunities for progress in our understanding of itinerant electron ferromagnetism presented by quantum Hall ferromagnets.
\end{abstract}

PACS numbers: 73.40.Hm, 75.10.Lp 
The ground state of a two-dimensional electron gas (2DEG) in the quantum Hall regime at Landau level filling factor $\nu=1$ is a strong ferromagnet with total spin quantum number $S=N / 2$. (Here $\nu \equiv N / N_{\phi}$ is the ratio of the number of electrons to the orbital degeneracy of a Landau level; $N_{\phi}=A B / \Phi_{0}=A /\left(2 \pi l_{c}^{2}\right)$ where $A$ is the area of the system, $\Phi_{0}$ is the magnetic flux quantum, and B is the magnetic field strength.) Recent NMR studies [1] of this system have provided evidence for the existence of electrically and topologically charged 11 Skyrmion excitations in the ground state for $\nu \neq 1$, as predicted by earlier theoretical work [2]. These observations have motivated further experimental studies of Skyrmions based on transport [3] and optical [4] measurements. Here [5] we address the accurate NMR measurements [1] of the temperature dependence of the spin magnetization, $M(T)$ at $\nu=1$. These data present an important challenge to theory since, as we explain at greater length below, their explanation requires a consistent treatment of fermion-quasiparticle and collective-magnetization excitations of the ferromagnetic ground state. The difficulties posed by this necessity have, for nearly seventy years, confounded attempts to develop the early work of Bloch [6] into a definitive theory of itinerant electron magnetism [7]. The existence of ferromagnetism in two-dimensional electron systems provides an important opportunity for progress, since theory does not need to contend with complicated band structures which have no essential importance but frustrate attempts to compare even relatively simple theoretical approximations with experiment. In this Letter we report on a theory for $M(T)$ which employs the simplest credible approximation in a many-body perturbation theory approach [8]. The absence of band-structure complications allows many steps in the calculation to be completed analytically and some relationships between the fermion-quasiparticle and collective pictures of the magnetization to be clearly established. In particular we show explicitly (for the first time as far as we are aware) that the magnetization suppression due to thermally excited spin-waves, which dominates in some limits, appears in a fermionparticle description as a reduction in the spectral weight of the quasiparticle pole. 
The microscopic Hamiltonian for the system in question is:

$$
\begin{aligned}
H & =-\frac{1}{2} \Delta_{z}\left(N_{\uparrow}-N_{\downarrow}\right)+\frac{1}{2} \frac{e^{2}}{\left(4 \pi \epsilon l_{c}\right)} \times \\
& \times \sum_{\substack{p, p^{\prime}, q \neq 0 \\
\sigma, \sigma^{\prime}}} \tilde{W}\left(q, p-p^{\prime}\right) c_{p+\frac{q}{2}, \sigma^{\dagger}}^{\dagger} c_{p^{\prime}-\frac{q}{2}, \sigma^{\prime}}^{\dagger} c_{p^{\prime}+\frac{q}{2}, \sigma^{\prime}} c_{p-\frac{q}{2}, \sigma}
\end{aligned}
$$

Here, the $c_{k, \sigma}^{(\dagger)}$ is a fermion creation operator for the lowest Landau level in a Landau gauge, (we choose $\sigma=\uparrow$ as the spin majority direction) and $N_{\sigma}$ is the number operator for spin $\sigma$. The two-particle matrix element for an isotropic interaction projected onto the lowest Landau level is

$$
\tilde{W}\left(q, p-p^{\prime}\right)=\int \frac{d^{2} \vec{k}}{(2 \pi)^{2}} \tilde{V}(\vec{k}) e^{-\frac{k^{2}}{2}} e^{i k_{x}\left(p-p^{\prime}\right)} \delta_{k_{y}, q}
$$

where $\tilde{V}(\vec{k})$ is the electron-electron interaction. We emphasize that this Hamiltonian is exact apart from corrections due to Landau level mixing, which become unimportant at strong magnetic fields.

The two energy scales in $H$ are the Zeeman energy $\Delta_{z} \equiv\left|g \mu_{B} B\right|$ and the electron-electron interaction energy scale $\lambda=e^{2} /\left(4 \pi \epsilon l_{c}\right)$. The ratio of these energy scales in the NMR [1] experiments $(\nu=1$ at $B=7 T), \Delta_{z} / \lambda \simeq 2.2 K / 136 K=0.016$, is small. Because of this fortunate experimental fact, Zeeman coupling to the spins acts like a weak symmetry breaking field even though we are in the strong magnetic field limit for the electron's orbital degrees of freedom. With the Zeeman field the ground state, denoted by $\mid 0>$, is the nondegenerate $S_{z}=N / 2$ state with all spin $\uparrow$ one-particle orbitals occupied [9]. As in the case of a localizedelectron ferromagnet the single spin-wave states (i.e., the eigenstates with $S_{z}=N / 2-1$ ) of this system can be determined exactly: [10,11, 13] $\left|\vec{k}>=1 / \sqrt{N} \sum_{q} e^{i q k_{x}} c_{q, \downarrow}^{\dagger} c_{q-k_{y}, \uparrow}\right| 0>$ and $\epsilon_{S W}(\vec{k})=\Delta_{z}+\lambda(\tilde{a}(0)-\tilde{a}(\vec{k}))$. (Here, $\tilde{a}(\vec{k})=\int\left(d^{2} \vec{q} /(2 \pi)^{2}\right) \tilde{V}(\vec{q}) e^{-q^{2} / 2} e^{i \vec{q} \cdot \vec{k}}$.) Because of the itinerant character of the electrons the wavevectors labelling the spin-wave states are not restricted to a Brillouin-zone; Pauli blocking of the electronic orbital degrees of freedom becomes less and less restrictive as more spins flip. The main importance of the Zeeman coupling, as detailed below, is to introduce a spin-wave gap and therefore cutoff their mag- 
netization suppression so that $M$ is finite at $T \neq 0$. The challenges in calculating $M(T)$ closely parallel [12] those for any itinerant electron ferromagnet [7].

A useful point of reference is the Hartree-Fock approximation (HFA), [6], which is especially simple in the quantum Hall regime [14]. This is the analog for quantum Hall systems of the band theory of itinerant electron magnetism. The HF orbital energies measured from the chemical potential solve the equation $\xi_{\downarrow}^{H F}=-\xi_{\uparrow}^{H F}=\left(\Delta_{z} / 2+\lambda \tilde{a}(0)\left(\nu_{\uparrow}^{H F}-1 / 2\right)\right)$ where $\nu_{\uparrow}^{H F}=n_{F}\left(\xi_{\uparrow}^{H F}\right)$. The solutions are plotted in Fig. 1. The 'exchange enhanced' spin-splitting of the HFA and the associated enhancement of the spin-polarization over its single particle value persist to high-temperatures. The sharp inflection points in Fig. 1 are a remnant of the spontaneous $\left(\Delta_{z}=0\right)$ magnetization which occurs incorrectly in the HFA for $k_{B} T<k_{B} T_{c}^{H F} \equiv \tilde{a}(0) / 4$. The well known failure of HF theory for itinerant electron ferromagnets [7] is particularly stark in the present case.

The weakness of the HFA rests in its inability to account for collective excitations of the ferromagnet which play a dominant role at low temperatures if $\Delta_{z}$ is small. In a manybody perturbation theory approach the simplest approximation which reflects the presence of spin-wave excitations in itinerant ferromagnets is one which includes a self-energy insertion consisting of a ladder sum of repeated interactions between HF electrons of one spin and holes of the opposite spin. The corresponding approximation has been discussed previously in theories of Hubbard model systems [15]. In the case of quantum Hall ferromagnets the sum may be evaluated explicitly and we find for the majority spin [16]:

$$
\begin{aligned}
& \Sigma_{\uparrow}\left(i \omega_{n}\right)=\lambda^{2}\left(\nu_{\uparrow}^{H F}-\nu_{\downarrow}^{H F}\right) \times \\
\times & \int_{0}^{\infty} d\left(\frac{k^{2}}{2}\right) \tilde{a}^{2}(k) \frac{\left.\left\{n_{B}\left(\tilde{\epsilon}_{S W}(k)\right)+\nu_{\downarrow}^{H F}\right)\right\}}{\left(i \hbar \omega_{n}+\tilde{\epsilon}_{S W}(k)-\xi_{\downarrow}^{H F}\right)} .
\end{aligned}
$$

A similar result is obtained for the minority spin. In Eq. (3) $n_{B}(\tilde{\epsilon}(k))$ is the Bose-Einstein distribution function and

$$
\tilde{\epsilon}_{S W}(\vec{k})=\Delta_{z}+\lambda\left(\nu_{\uparrow}^{H F}-\nu_{\downarrow}^{H F}\right)(\tilde{a}(0)-\tilde{a}(\vec{k}))
$$

is the finite-temperature spin-wave dispersion in this approximation. This self-energy describes the emission and absorption of a virtual spin-wave; $\tilde{a}(k)$ which describes the spin-- 
wave dispersion is, significantly as we discuss below, also the coupling constant for the interaction of electrons with spin-waves.

After analytical continuation $\left(i \hbar \omega_{n} \rightarrow \hbar \omega+i \eta\right)$ we see that the $\Sigma_{\uparrow}^{r e t}$ is complex in the interval $I \equiv\left(\xi_{\uparrow}^{H F}, \xi_{\downarrow}^{H F}-\Delta_{z}\right)$, where real transitions with electronic spin flips and spin-wave absorption are possible, and is real outside $I$. The real part of $\Sigma_{\uparrow}^{r e t}$ diverges to $-\infty$ at the lower limit of $I$ and to $+\infty$ at its upper limit so that the retarded Green's function $G_{\uparrow}^{r e t}(\omega)=\left(\hbar \omega-\xi_{\uparrow}^{H F}-\Sigma_{\uparrow}^{r e t}(\omega)\right)^{-1}$ has a branch cut along $I$ and poles on opposite sides at $\omega_{ \pm}^{*}$. Note that $\Sigma_{\uparrow}^{r e t}$ vanishes and the $\mathrm{HF}$ results are correctly recovered for $T \rightarrow 0$. For $T \rightarrow \infty \sum_{\uparrow}^{r e t}$ again vanishes because $\nu_{\uparrow}^{H F}-\nu_{\downarrow}^{H F}$ vanishes. In the intermediate temperature region corrections to the HFA are important.

To compare with the NMR $M(T)$ measurements we evaluate the spin magnetization from $A_{\uparrow}(\omega)=-2 \operatorname{Im} G_{\uparrow}^{r e t}(\omega)$, using $M(T)=\left(M_{0} / \nu\right) \int(d \omega / 2 \pi) n_{F}(\omega)\left(A_{\uparrow}(\omega)-A_{\downarrow}(\omega)\right)$ and $A_{\uparrow}(\omega)=A_{\downarrow}(-\omega)$. (The last identity is a consequence of particle-hole symmetry.) Results 17] for $A_{\uparrow}(\omega)$ are shown in Fig. 2 where we see that deviations from the single $\delta$ function peak of the HFA increase strongly with temperature. At low $T$ nearly all of the spectral weight is in the pole at $\omega_{-}^{*}$ which is shifted only slightly from $\xi_{\uparrow}^{H F}$. For $T \rightarrow 0$ we see from Eq. (3) that the residue of this pole is given by

$$
\begin{aligned}
z_{-} & \rightarrow 1+\frac{\partial \sum_{\uparrow}^{r e t}(\omega)}{\partial \omega}\left|\omega=\xi_{\uparrow}^{H F}\right| \\
& \rightarrow 1-\int_{0}^{\infty} d\left(\frac{k^{2}}{2}\right) n_{B}\left(\tilde{\epsilon}_{S W}(k)\right) .
\end{aligned}
$$

In the last form of Eq. (5) we have set $\nu_{\downarrow}^{H F}=0$ for $T \rightarrow 0$. Note that $\tilde{a}(k)$ cancels out of the final form of Eq.(5) because of the relationship between the coupling function and the spin-wave dispersion. From this result we see that the reduction in $M$ at low $T$ due to thermally excited spin-waves, familiar from continuum and Heisenberg type models of magnetism (see for example Ref. [8]) appears in a microscopic itinerant electron theory as a reduction in the quasiparticle normalization factor on going beyond a HFA.

Results for $M(T)$ calculated from these spectral functions for a set of parameters appropriate to the NMR experiments [1] are shown in Fig. 3 and compared with results from 
other theories. The HFA yields $\left.M(T) / M_{0}=2 n_{F}\left(\xi_{\uparrow}^{H F}\right)-1=\tanh \left(\beta \xi_{\downarrow}^{H F}(\beta) / 2\right)\right)$ and is exact both for $T \rightarrow 0$ and $T \rightarrow \infty$. As expected it grossly overestimates the observed magnetization. The present theory predicts a strong suppression of the magnetization at much lower temperatures because it includes the effects of thermally excited spin-waves. Our results are extremely insensitive to the choice of screening wavevector [17]. A more complete theory would have temperature and frequency dependent screening; the value $q_{s c}=0.01 l_{c}^{-1}$, is the [16 self-consistent static screening wavevector temperature in our theory for $T \simeq 0.09$. We observe that an excellent approximation to our results is obtained by including only the two poles in the spectral function. The two poles correspond respectively to the Hartree-Fock quasiparticle state and a state with a minority spin quasiparticle and a spin-wave. This suggests that at higher temperatures an improved theory would need to describe multiple spin-wave dressings of the fermion propagator. At low temperatures the reduction in $M(T)$ is dominated by the long-wavelength spin-wave contribution which gives $M(T) / M_{0}-1=C(T) T \ln \left(1-e^{-\Delta_{z} / k_{B} T}\right)$ where $C(T)$ depends weakly on temperature. This suppression crosses over from an activated temperature dependence $\left(-C(T) T e^{-\Delta_{z} / k_{B} T}\right)$ for $k_{B} T<\Delta_{z}$ to an approximately linear temperature dependence $\left(-C(T) T \ln \left(\Delta_{z} / k_{B} T\right)\right)$ for $k_{B} T>\Delta_{z}$ but still small. The linear $T$-dependence is the two-dimensional analog of the $T^{3 / 2}$-Bloch law familiar in three-dimensional ferromagnets 18.

To compare with experiment it is necessary to account for the finite thickness of the twodimensional electron layer by including form factors in determining the effective electronelectron interaction. We see in Fig. 3 that although our theory overestimates $M(T)$, the improvement compared to the HFA is considerable. One obvious shortcoming of the simple theory is the use of HFA propagators in calculating $\tilde{\epsilon}_{S W}(k)$; the fact that this quantity depends on the propagator only through $\nu_{\sigma}^{H F}$ suggests that the theory could be improved simply by replacing the HF filling factors by the those calculated including self-energy corrections. As shown in Fig. 3, applying this procedure leads to an further decrease of $M(T)$. Overall the agreement of this relatively simple theory with experiment gives hope that a fairly complete theory can be built from this starting point. It is possible that part 
of the discrepancy originates in the difficulty of precisely locating $\nu=1$ experimentally, [1], since small changes in filling factor are known to cause dramatic changes in the ground state magnetization. However, we believe that most of the discrepancy in Fig. 3 is due to limitations of the theory. The opportunity for such a direct comparison of theory and experiment should be helpful in developing a more complete theory. We believe that any lessons learned from advances in this direction will be transferable to all itinerant electron ferromagnets.

We thank S. Barrett for discussions and for sharing data from the NMR-measurements prior to publication. Helpful interactions with W. Apel, S. M. Girvin, C. Hanna, R. Haussmann and H. Mori are gratefully acknowledged. One of the authors (M.K.) is supported by a fellowship from the German Academic Exchange Service (DAAD). This work was supported in part by NSF grant No. DMR94-16906.

† Present address: Institut für Theoretische Physik, Universität des Saarlandes, PF 1511 50, D-66041 Saarbrücken, Germany. 


\section{REFERENCES}

[1] S. E. Barrett, R. Tycko, L. N. Pfeiffer, K. W. West, Phys. Rev. Lett. 72, 1368 (1994); R. Tycko, S. E. Barrett, G. Dabbagh, L. N. Pfeiffer, K. W. West, Science 268, 1460 (1995); S. E. Barrett, G. Dabbagh, L. N. Pfeiffer, K. W. West, R. Tycko, Phys. Rev. Lett. 74, 5112 (1995).

[2] D. H. Lee, C. L. Kane, Phys. Rev. Lett. 64, 1313 (1990); S. L. Sondhi, A. Karlhede, S. A. Kivelson, E. H. Rezayi, Phys. Rev. B47, 16419 (1993); H. A. Fertig, L. Brey, R. Côté, A. H. MacDonald, Phys. Rev. B 50, 11018 (1994).

[3] A. Schmeller, J.P.Eisenstein, L.N.Pfeiffer, K.W.West, preprint cond-mat/9506113, (1995).

[4] E.H.Aifer, B.B.Goldberg, D.A.Broido, preprint cond-mat/9507069, (1995).

[5] An account of a preliminary version of this work has been published previously: Marcus Kasner, A. H. MaDonald, Proceedings of the Workshop on Novel Physics in LowDimensional Electron Systems, Madras, 1995, Physica B 212, 289 (1995).

[6] F. Bloch, Z. Physik 57, 545 (1929).

[7] For reviews of theoretical work on itinerant electron ferromagnets see C. Herring in Magnetism, Volume 4, edited by G. T. Rado and H. Suhl, (Academic, New York, 1966) and T. Moriya, Spin Fluctuations in Itinerant Electron Magnetism (Springer-Verlag, Solid State Science, Vol. 56, Berlin, 1985).

[8] A complimentary theoretical approach has recently been taken by Read and Sachdev in a study which is also motivated by the NMR $M(T)$ data. These authors use a continuum quantum field theory model and focus on collective aspects of the physics: N.Read, S.Sachdev, preprint cond-mat/9507103, (1995).

[9] Kun Yang, K. Moon, L. Zheng, A. H. MacDonald, S. M. Girvin, D. Yoshioka, S.- 
C. Zhang, Phys. Rev. Lett. 72, 732 (1994); K. Moon, H. Mori, Kun Yang, S. M. Girvin, A. H. MacDonald, L. Zheng, D. Yoshioka, S.-C. Zhang, Phys. Rev. B51, 5138 (1995).

[10] Yu. A. Bychkov, S. V. Iordanskii, G. M. Eliashberg, Pis'ma Ž. Eksp. Teor. Fiz. 33, 152 (1981) (JETP Letters 33, 143 (1981)).

[11] C. Kallin, B. I. Halperin, Phys. Rev. B30, 5655 (1984).

[12] Quantum Hall ferromagnets do have the unusual feature of having a kinetic energy which is independent of the orbital state. Generalized Hubbard models with this 'flat-band' property have also been studied in connection with attempts to rigorously establish ferromagnetism in that class of models. The 'flat-band' limit of narrow-band Hubbard ferromagnets is not expected to be pathological. See Hal Tasaki, Phys. Rev. Lett. 73, 1158 (1994); ibid preprint, cond-mat/9509063, (1995). Disorder in quantum Hall systems plays a role similar to band dispersion in Hubbard models.

[13] M. Rasolt, A. H. MacDonald, Phys. Rev. B34, 5530 (1986).

[14] T. Ando, Y. Uemura, J. Phys. Soc. Japan, 37, 1044 (1974).

[15] See for example, J.A. Hertz and D.M. Edwards, J. Phys. F 3, 2174 (1973).

[16] A detailed description of this calculation will be published later.

[17] Becauase of the long-range of the Coulomb interaction the self-energy has a weak logarithmic divergence due to the absorption of very short wavelength spin-waves. This divergence is completely removed by any amount of the screening which must be present in a more complete theory. For the numerical results shown here we introduced a small ad hoc screening wavevector with magnitude $q_{s c}=0.01 l_{c}^{-1}$ except where noted.

[18] See e. g. D. C. Mattis, The Theory of Magnetism II, Thermodynamics and Statistical Mechanics (Springer-Verlag, Solid State Science, Vol. 55, Berlin, 1985). 


\section{Figure captions:}

Fig. 1: HFA orbital energies $\xi_{\sigma}^{H F}$ in units of $\lambda$ as a function of temperature for Coulombic electron-electron interactions and $\nu=1$. The solid line is $\xi_{\uparrow}^{H F}$ and the long-dashed line is

$\xi_{\downarrow}^{H F}$. These results are for $\Delta_{z}=0.016$. The HFA spin magnetization is $M=M_{0}\left(\nu_{\uparrow}^{H F}-\nu_{\downarrow}^{H F}\right)$ where $M_{0}=\left(\left|g \mu_{B}\right| / 2\right) N$ is the total magnetization at $T=0$.

Fig. 2: The spectral function $A_{\uparrow}(\omega)$ for parameter values $\Delta_{z}=0.016$ and $q_{s c} l_{c}=0.01$ at temperatures $T=0.05,0.1$ and 0.2 in units of $\lambda / k_{B}\left(\Delta_{z}\right.$ and $\omega$ are in units of $\left.\lambda\right)$. The numbers indicate the fraction of the total spectral weight from the two poles and from the branch cut.

Fig. 3: Results for $M(T)$ for $\Delta_{z}=0.016$. The long-dashed and short-dashed lines show the results of the present theory with two-different choices for the ad hoc screening vector, $q_{s c}=0.01 l_{c}^{-1}$ and $q_{s c}=0.1 l_{c}^{-1}$. Results incorporating a finite quantum well width $\left(w=30 n m \simeq 3.11 l_{c}\right)$ with $q_{s c}=0.01 l_{c}^{-1}$ are shown as a solid line. The experimental results of Barrett et al. are shown as crosses. For comparison, the dot-dashed curve is the result of the SC-HFA $\left(q_{s c}=0.01 l_{c}^{-1}\right)$ theory and the solid curve with stars shows the magnetization obtained when the spin-wave dispersion is calculated from self-consistently determined partial filling factors. 


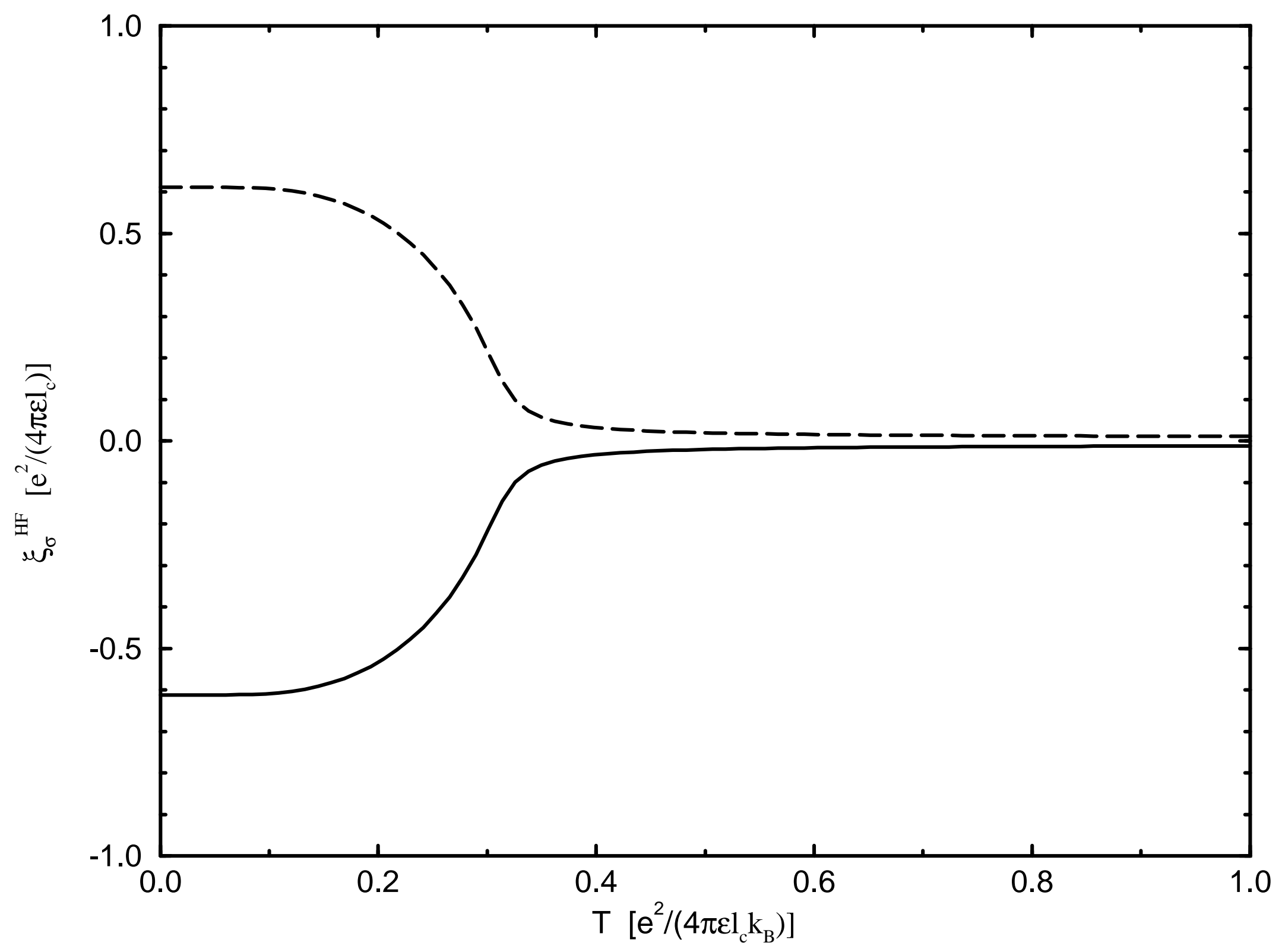



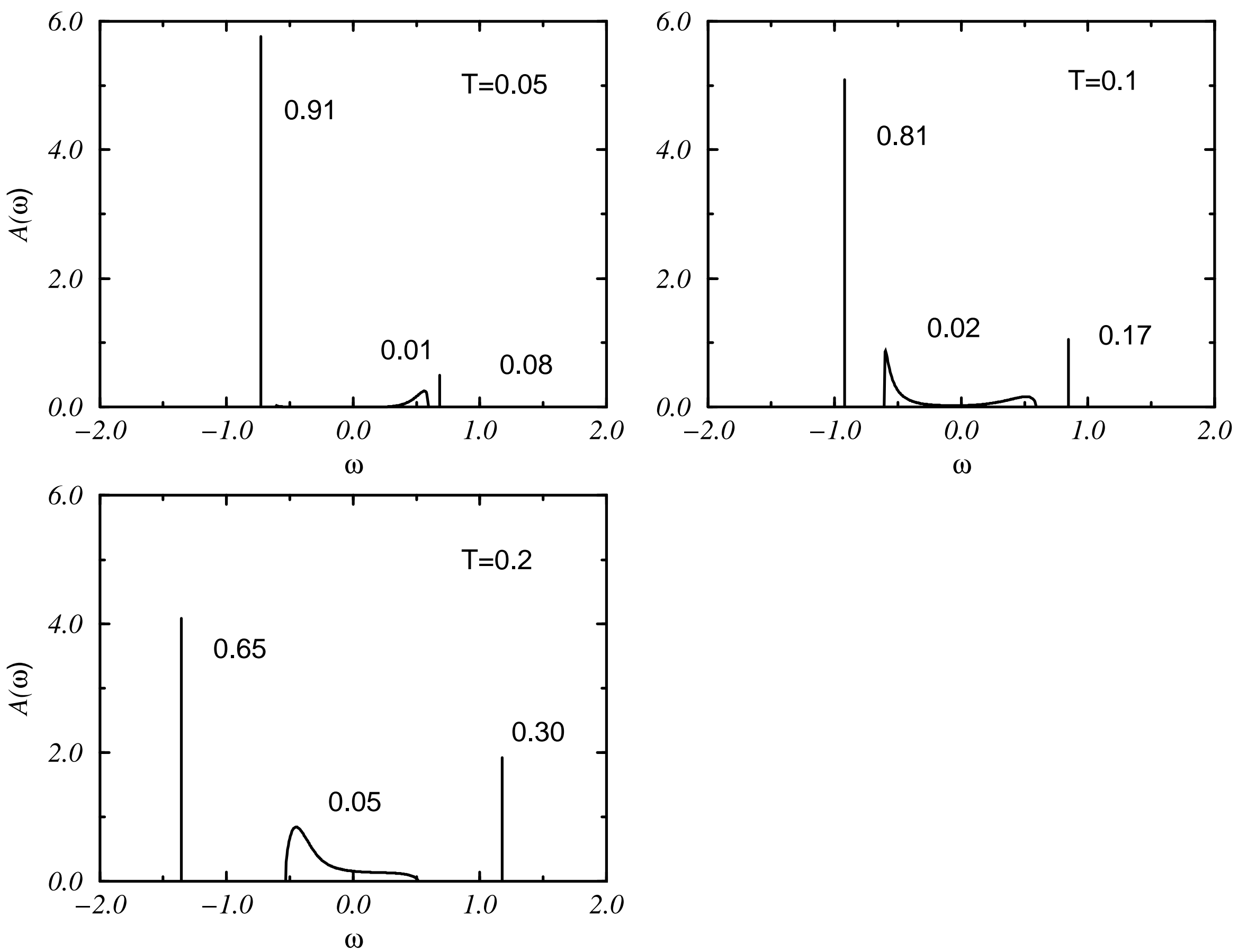


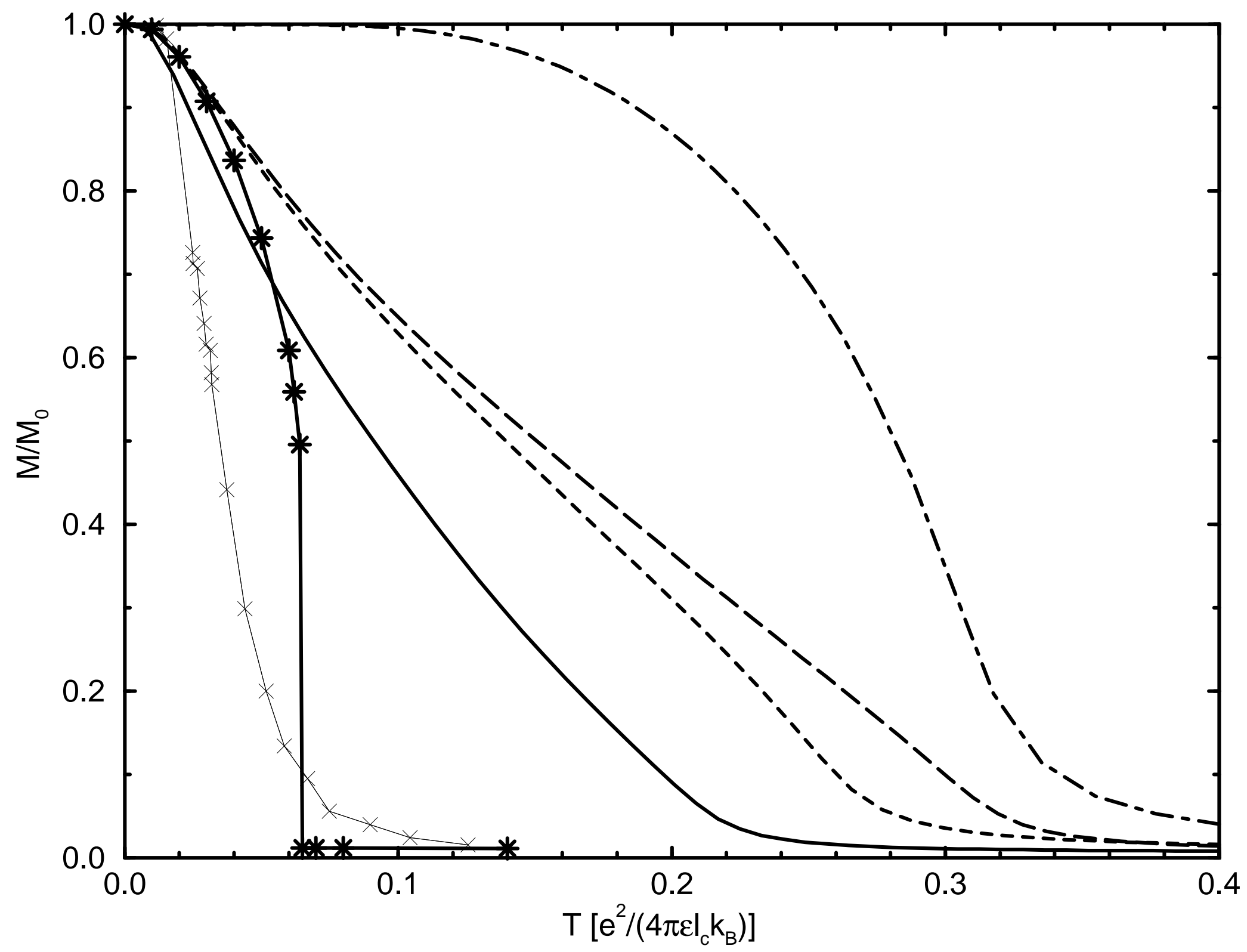

